\title{
Proton Irradiation of e2v technologies L3Vision Devices
}

\author{
D. R. Smith ${ }^{\dagger}$, A. D. Holland
}

Imaging for Space and Terrestrial Applications Group, School of Engineering and Design, Brunel University, Uxbridge, Middlesex, UB8 3PH, UK.

\begin{abstract}
This paper describes the proton irradiation and subsequent analysis of 8 e2v technologies CCD65 L3Vision devices with the intention of assessing the suitability of L3Vision technology to applications in space. In particular the use of L3Vision charge coupled devices (CCDs) on the Radial Velocity Spectrometer (RVS) instrument of the planned European Space Agency GAIA mission is discussed. The operational properties of the devices were characterised before irradiation with protons to a $10 \mathrm{MeV}$ equivalent fluence of $2.5 \times 10^{9}$ protons $\mathrm{cm}^{-2}$. The devices were then characterised again before undergoing a second proton irradiation which increased the total $10 \mathrm{MeV}$ equivalent proton fluence received by each device to $2.1 \times 10^{10}$ protons $\mathrm{cm}^{-2}$. All 8 devices functioned as expected after each irradiation, with no catastrophic failures occurring even after the second irradiation which increased the total proton fluence to approximately 10 times the expected 6 year RVS fluence.
\end{abstract}

Key Words: CCD, charge coupled device, radiation damage, proton irradiation, L3Vision

\section{Introduction}

For any new CCD technology being considered for application in space, it is necessary to asses the impact of proton irradiation on the operational characteristics of devices featuring the technology. L3Vision is one such technology, as it may be used on the Radial Velocity Spectrometer (RVS) instrument that forms part of the European Space Agency GAIA mission [1]. An earlier proton irradiation study of two e2v technologies L3Vision devices [2, 3] revealed no significant changes in the operating characteristics of the devices after irradiation but concluded that a further study was required to determine if emission sites generated in the high field regions of the gain register pixels could cause device failure. A study to irradiate an increased number of devices to higher proton fluence was then initiated to improve statistics on the likelihood of device failure due to proton irradiation. This paper first describes the L3Vision technology and the irradiation methodology before presenting a detailed analysis of 8 devices before and after two separate proton irradiations. The first irradiation was to a $10 \mathrm{MeV}$ equivalent fluence of $2.5 \times 10^{9}$ protons $\mathrm{cm}^{-2}$ and the second irradiation increased the total fluence received by each device to $2.1 \times 10^{10}$ protons $\mathrm{cm}^{-2}$, approximately 10 times the RVS 6 year expected fluence [4].

\footnotetext{
${ }^{\dagger}$ Corresponding author, Dr David Ryan Smith, ISTA, School of Engineering and Design, Brunel University, Uxbridge, Middlesex, UB8 3PH, UK; phone +44 (0) 189 5266593, fax +44 (0) 189 5258728, email david.smith@brunel.ac.uk.
} 


\section{The e2v technologies L3Vision CCD65}

The e2v technologies Low Light Level (L3Vision) CCD uses a novel method of charge readout that is capable of an equivalent output noise of less than one electron at pixel rates of over $11 \mathrm{MHz}$ [5, 6]. The CCD is an inverted mode operation frame transfer device that has a standard readout register followed by a 'gain' register that multiplies the signal charge before it is converted to a voltage. The image and store sections of the CCD are each 591 pixels $\times 296$ pixels, while the readout and gain registers are each 591 pixels in length plus a few reference pixels. Figure 1 shows a photograph of an L3Vision CCD65 device and Figure 2 shows the device geometry. The pixels in the image, store and readout register of the device measure $20 \mu \mathrm{m} \times 30 \mu \mathrm{m}$, while pixels in the gain register measure $40 \mu \mathrm{m} \times 30 \mu \mathrm{m}$ to handle the potentially larger signal charge after gain. The device parameters are summarised in Table 1.

The gain in the signal arises as charge is passed through the gain register of the L3Vision device. One electrode in each of the pixels in the register, $\phi \mathrm{HV}$, is clocked with a much higher voltage than is needed to simply transfer the charge. An additional electrode held at 2 volts d.c. is included immediately before the high voltage electrode which is typically held at $\sim 40$ - 50 volts. The large electric field between the high voltage electrode and the d.c. electrode causes the charge carriers to be accelerated to a high enough velocity to generate more charge carriers through the process of impact ionisation [7].

The average gain per pixel transfer through the gain register is generally small, but on passing through the full 591 gain register elements the total gain approaches a few hundred. If a gain of unity is selected, the L3Vision device operates in the same way as a standard device, with a single extra row in the readout sequence. The measured variation in gain with applied bias voltage at three different temperatures is shown in Figure 3. The gain is seen to increase with decreasing temperature at a given applied voltage due to the temperature dependence of the electron ionisation rate. For a given electric field, the ionisation rate increases with decreasing temperature [8].

\section{Irradiation Methodology}

The 8 L3Vision CCD65 devices were irradiated using the proton accelerator facility at the Paul Scherrer Institut in November 2003 and June 2004. The devices were irradiated in two batches of four and no shielding was used. The energy of the proton beam for the first irradiation run was $63 \mathrm{MeV}$ and the proton dose given to each device was $5.8 \times 10^{9}$ protons $\mathrm{cm}^{-2}$. According to the Non-Ionising Energy Loss (NIEL) damage relationship, the $10 \mathrm{MeV}$ equivalent fluence was therefore $\sim 2.5 \times 10^{9}$ protons $\mathrm{cm}^{-2}$. The energy of the proton beam for the second irradiation run in 2004 was reduced to $10 \mathrm{MeV}$. The proton fluence given to each device in this case was $1.8 \times 10^{10}$ protons $\mathrm{cm}^{-2}$, increasing the total $10 \mathrm{MeV}$ equivalent fluence received by each device to $2.1 \times 10^{10}$ protons $\mathrm{cm}^{-2}$. Table 2 lists the device numbers, the $10 \mathrm{MeV}$ equivalent proton fluence received after the first and second proton irradiations, the time taken for each irradiation and the total $10 \mathrm{MeV}$ equivalent proton dose received by each device. Figure 4 shows the layout of a single batch of 4 CCDs during irradiation. The CCDs were located $32 \mathrm{~cm}$ from the end of the proton beam line. Each batch was irradiated using the same geometry as presented in the figure. The beam uniformity was measured to be within $10 \%$ over a $9 \mathrm{~cm}$ diameter centred in the middle of the 4 CCDs. The beam uniformity, beam calibration measurements and irradiation of the devices were carried out by PSI staff. 


\section{Device Characterisation Method}

The CCDs were each tested prior to irradiation to ensure they worked correctly and exhibited no unusual operating characteristics. The same set of clock voltages and delays were used for each device. The potential applied to the high voltage electrode, $\phi \mathrm{HV}$, was $27.5 \mathrm{~V}$, giving an output signal gain of $\sim 2$. This setting was used to characterise the cosmetic quality of the readout and gain registers rather than to test the L3Vision gain. All 20 devices operated within usual parameters in dark conditions at room temperature.

A novel sequencer program was written and used to characterise the readout and gain registers of a given device. The sequencer reads out only the readout and gain registers of each device in order to identify any bright defects generated within these regions. The image clocks are suspended during readout to avoid any thermal leakage current from the image and store sections entering into the readout register. A short series of $3 \mathrm{~ms}$ row integrations are then taken and a pseudo-image of the variation in charge level in each pixel of the readout and gain registers over time is created. An example of such an image is given in Figure 5. The figure includes diagrams of a CCD65 device to correlate the sections of the recorded image with the physical sections of the device.

Figure 6 depicts the expected line profile for the registers when clocked-out in this mode. The dark current contributions from the various sections are labelled. The signal in the gain register increases as a function of the number of transfers, representing a constant leakage current per pixel, but with increasing gain along the register. However, the signal in the readout register is uniform since all of these pixels have roughly the same leakage current and all go through the same number of gain elements. Note that the leakage current per pixel in the readout register is approximately half that in the gain register because the pixels occupy half the physical area.

\section{Device Characterisation}

\subsection{Bright Pixels}

The 8 devices were tested after each irradiation in dark conditions at room temperature. After the first irradiation, a readout and gain register line profile (the average of 100 image rows in each case) for each device was recorded with, the high voltage clock, $\phi \mathrm{HV}$, set to $40.0 \mathrm{~V}$. After the second irradiation a similar data set was recorded (using the same operational voltages) for direct comparison. However, due to the increased dark signal generated as a result of the second irradiation, it was also necessary to take further data sets after reducing $\mathrm{V}_{\text {od }}$ from $28.9 \mathrm{~V}$ to $27.9 \mathrm{~V}$ so as to give a better full ADC scale spread in the data points and prevent ADC saturation. Three data sets were collected while using the reduced $\mathrm{V}_{\text {od }}$ setting with $\phi \mathrm{HV}$ set at $27.5 \mathrm{~V}, 37.5 \mathrm{~V}$ and $40.5 \mathrm{~V}$ respectively.

Data from an unirradiated CCD65 device of the same batch (00463-10-14) was also taken at the same time as the other data sets, the device acting as a control for comparison of unirradiated and irradiated line profiles. All the data presented are normalised to the overscan pixel level indicated in Figure 6. 
Figure 7 shows the unirradiated CCD65 data and the data collected after each PSI irradiation for device 00463-07-26. The data shown as dashed lines were taken from the unirradiated control device, the data in solid lines are the direct comparison of data taken after each of the two irradiations at $40.0 \mathrm{~V}$ and the data in dotted lines are data collected using the updated voltage and sequencer settings. Figure 8 compares the $40.5 \mathrm{~V}$ data from each of the 8 devices after the second irradiation. The figure demonstrates the gain function is well behaved at $40.5 \mathrm{~V}$, which is significant as a $\phi \mathrm{HV}$ value of this magnitude gives the required gain of $\sim 10$ proposed for the GAIA RVS instrument. The RVS instrument uses a standard low noise amplifier with $\sim 5$ - 7 electrons rms at $240 \mathrm{kHz}$ and the gain of 10 reduces the equivalent noise charge to $<1$ electron rms.

All devices remained operational after irradiation to $2.1 \times 10^{10}$ protons $\mathrm{cm}^{-2}$. This result is highly significant since the proton fluence used in the first irradiation, $2.5 \times 10^{9}$ protons $\mathrm{cm}^{-2}$, is of a similar magnitude to the expected end of life proton fluence expected for space missions located at L2, such as GAIA, and the proton fluence received after the second irradiation, $2.1 \times 10^{10}$ protons $\mathrm{cm}^{-2}$, is equivalent to testing $\sim 5 \mathrm{RVS}$ focal planes to the expected end of life mission fluence. These results build strong confidence in the use of L3Vision technology for space instrumentation.

The spread in line profile from device to device is due largely to inter-CCD variance rather than any slight fluctuations in the irradiation dosimetry. After irradiation, all devices exhibited increased noise and had bright defects in both the readout and gain registers. The increase in dark current and number of bright pixels generated after each irradiation are comparable to previous proton irradiation studies on other e2v technologies CCDs [9, 10]. The largest dark current spikes are seen to occur in the gain register. Figure 9 shows histograms of the dark current spike amplitudes in both the gain register and the normal readout register of all the devices. The spike amplitudes in the gain register were normalised for gain by subtracting a linear baseline fit to the data. In general the derived spike distributions in the gain and readout registers are similar, the 3 - 4 brightest spikes most likely being due to Frenkel-Pool emission [11] in the higher-field regions of the gain register. At least one bright pixel was observed to exhibit random telegraph signal (RTS) behaviour, the charge level alternating between two distinct levels over time [12, 13].

\subsection{Charge Smearing}

Evidence of charge smearing in the readout direction after the $2.1 \times 10^{10}$ protons $\mathrm{cm}^{-2}$ irradiation can be seen when looking at bright pixels generated in the readout register of a device. Figure 10 shows an example of charge smearing in the readout register of device 00463-07-08. The bright pixel was generated as a result of the second PSI proton irradiation (the bright pixel was certainly not present in the data taken after the first irradiation) and trailing charge can be seen in the few pixels following the bright defect.

\section{Conclusions}

This study considered 8 e2v L3Vision CCD65 devices that were exposed them to two levels of proton irradiation. The first irradiation to a $10 \mathrm{MeV}$ equivalent proton fluence of $\sim 2.5 \times 10^{9}$ protons $\mathrm{cm}^{-2}$ is $\sim 5$ times the anticipated mission fluence of the XMM-Newton European Photon Imaging Camera detectors [14] and is similar to that expected for 
detectors on the GAIA mission [4]. After the first irradiation, all 8 devices functioned within normal parameters and exhibited comparable numbers of bright pixels in both the gain and register elements as have been found in other proton irradiation studies of CCDs $[9,10]$.

Statistical analysis of bright pixel amplitudes in the gain and normal register identified that the distribution of bright pixels in the gain register was the same as that in the normal register. However, it should also be noted that at least 3 of the brightest pixels occurred in the gain register, which may indicate enhanced emission from the higher fields over a limited area in these pixels.

After the second proton irradiation, increasing the total $10 \mathrm{MeV}$ equivalent fluence to $2.1 \times 10^{10}$ protons $\mathrm{cm}^{-2}$ (approximately 10 times the RVS 6 year expected fluence) all 8 devices functioned with no catastrophic failures. This study is equivalent to testing $~ 5$ RVS focal planes to the expected end of life mission fluence and indicates that the risk of catastrophic failure of any of the devices is low.

Further to this we conclude that most of the pixels in the devices contain bright defects after irradiation to a level of $2.1 \times 10^{10}$ protons $\mathrm{cm}^{-2}$. In the majority of cases, bright pixels generated in the readout and gain registers of the devices become shallower at higher gain, the flat-field level increasing more than the bright pixel amplitude. At least one of the bright pixels exhibited random telegraph signal (RTS) behaviour [12, 13], which is not unexpected and the fluctuation would contribute a negligible variation to the average leakage current generated in the gain register. The bright pixel amplitude is unaffected by fluence while the dark current is seen to increase accordingly. The increase in dark current seen after the first proton irradiation and then after the second irradiation is comparable from device to device.

Evidence of charge smearing after the $2.1 \times 10^{10}$ protons $\mathrm{cm}^{-2}$ irradiation can be seen when looking at bright pixels generated in the readout register of a device. Trailing charge is observed in $\sim 4-6$ pixels in the across scan direction following the bright pixel. This affects RVS sensitivity and we have currently set an end of life proton fluence target of $2 \times 10^{9}$ protons $\mathrm{cm}^{-2}$.

Further work will involve a more detailed investigation of the gain vs. $\phi H V$ clock bias in the irradiated devices to examine the impact on specific space instruments. The gain characteristics as a function of temperature will be explored, in particular, the extension of the post-irradiation characterisation to cryogenic temperatures, over the range $-120^{\circ} \mathrm{C}$ to room temperature, to ensure that the additional leakage current from the gain register "freezes out" at the application temperature.

\section{Acknowledgements}

The authors would like to thank Peter Pool and Ralph Holtom of e2v technologies for the provision of the test devices and for comments on the work. Thanks also to e2v and PSI staff for helping with the proton irradiations. 


\section{References}

[1] A. D. Holland, M. Cropper, D. Katz, I. B. Hutchinson, R. M. Ambrosi, T. Stevenson, D. R. Smith, D. Walton, D. Burt, P. Pool, D. Morris, T. Paulsen, “CCDs for the Radial Velocity Spectrometer on GAIA”, Proc. SPIE, vol. 5251, (2004), pp. 253-60.

[2] D. R. Smith, A. D. Holland, M. S. Robbins, “The effect of protons on E2V Technologies L3Vision CCDs”, Nuc. Inst. Meth., vol. A513, (2003), pp. 296-99.

[3] D. R. Smith, "Radiation Damage in Charge Coupled Devices”, Ph.D. thesis, University of Leicester, $12^{\text {th }}$ September 2003.

[4] H. Evans, “GAIA Radiation Analysis: Cornerstone Launch in 2010, 6 yr Extended Mission”, ESA report, esa/estec/ema/he/GAIA/3b, (2003).

[5] P. Jerram, P. Pool, R. Bell, D. Burt, S. Bowring, S. Spencer, M. Hazelwood, I. Moody, N. Catlett, P. Heyes, “The LLLCCD: Low Light Imaging without the need for an intensifier”, Proc. SPIE, vol. 4306, (2001).

[6] C. D. Mackay, R. N. Tubbs, R. Bell, D. Burt, P. Jerram, I. Moody, "Sub-Electron Read Noise at MHz Pixel Rates”, Proc. SPIE, vol. 4306, (2001), pp. 289-98.

[7] Grove, A. S., "Physics and Technology of Semiconductor Devices”, John Wiley and Sons Inc., 1967.

[8] Sze, S. M., “Physics of Semiconductor Devices”, John Wiley and Sons Ltd., $2^{\text {nd }}$ Ed., 1981.

[9] D. R. Smith, A. D. Holland, M. S. Robbins, R. M. Ambrosi, I. B. Hutchinson, "Proton induced leakage current in CCDs”, Proc. SPIE, vol. 4851, (2003), pp. 842-48.

[10] R. M. Ambrosi, A. D. T. Short, A. F. Abbey, A. A. Wells, D. R. Smith, "The effect of proton damage on the X-ray spectral response of MOS CCDs for the Swift X-ray Telescope”, Nuc. Inst. Meth., vol. A482, (2002), pp. 644-52.

[11] Martin, P. A., B. G. Streetman, K. Hess, “Electric field enhanced emission from non-coulombic traps in semiconductors”, J. Appl. Phys., vol. 52, no. 12, (1981), pp. 7409-15.

[12] D. R. Smith, A. D. Holland, M. S. Robbins, R. M. Ambrosi, I. B. Hutchinson, "Proton induced leakage current in CCDs”, Proc. SPIE, vol. 4851, (2003), pp. 842-48.

[13] D. R. Smith, A. D. Holland, I. B. Hutchinson, “Random Telegraph Signals in Charge Coupled Devices”, Nuc. Inst. Meth., vol. A530, (2004), 521-35.

[14] Holmes-Siedle, A. G., S. J. Watts, A. D. Holland, “The irradiation, measurement and technology evaluation of X-ray sensitive CCDs for ESA scientific missions”, Final report on ESTEC contract no. 8815/90/NL/LC(SC), Brunel University, Uxbridge, UB8 3PH, UK, (1995). 


\begin{tabular}{|c|c|c|}
\hline \multicolumn{2}{|l|}{ Parameter } & Value \\
\hline \multicolumn{2}{|c|}{ Active image area } & $11.52 \times 8.64 \mathrm{~mm}$ \\
\hline \multicolumn{2}{|c|}{ Image section } & $591 \times 296$ pixels \\
\hline \multicolumn{2}{|c|}{ Store section } & $591 \times 296$ pixels \\
\hline \multirow{4}{*}{ Pixel size: } & Image section & $20 \times 30 \mu \mathrm{m}$ \\
\hline & Store section & $13.5 \times 30 \mu \mathrm{m}$ \\
\hline & Readout register & $20 \times 30 \mu \mathrm{m}$ \\
\hline & Gain register & $40 \times 30 \mu \mathrm{m}$ \\
\hline \multicolumn{2}{|c|}{ Spectral range } & $400-1060 \mathrm{~nm}$ \\
\hline
\end{tabular}

Table 1

\begin{tabular}{|c|c|c|c|c|c|}
\hline \multirow{2}{*}{$\begin{array}{l}\text { Device } \\
\text { Number }\end{array}$} & \multicolumn{2}{|c|}{ First Irradiation } & \multicolumn{2}{|c|}{ Second Irradiation } & \multirow{2}{*}{$\begin{array}{c}\text { Total } 10 \mathrm{MeV} \\
\text { equivalent fluence } \\
\text { received } \\
\text { (protons } \mathrm{cm}^{-2} \text { ) }\end{array}$} \\
\hline & $\begin{array}{c}10 \mathrm{MeV} \\
\text { equivalent } \\
\text { fluence } \\
\text { (protons } \mathrm{cm}^{-2} \text { ) }\end{array}$ & $\begin{array}{l}\text { Time for } \\
\text { irradiation } \\
\text { (seconds) }\end{array}$ & $\begin{array}{c}10 \mathrm{MeV} \\
\text { equivalent } \\
\text { fluence } \\
\text { (protons } \mathrm{cm}^{-2} \text { ) }\end{array}$ & $\begin{array}{l}\text { Time for } \\
\text { irradiation } \\
\text { (seconds) }\end{array}$ & \\
\hline 00463-07-08 & $2.54 \times 10^{9}$ & 73 & $1.81 \times 10^{10}$ & 268 & $2.1 \times 10^{10}$ \\
\hline 00463-07-11 & $2.55 \times 10^{9}$ & 90 & $1.81 \times 10^{10}$ & 277 & $2.1 \times 10^{10}$ \\
\hline 00463-07-15 & $2.55 \times 10^{9}$ & 90 & $1.82 \times 10^{10}$ & 271 & $2.1 \times 10^{10}$ \\
\hline 00463-07-26 & $2.51 \times 10^{9}$ & 578 & $1.82 \times 10^{10}$ & 271 & $2.1 \times 10^{10}$ \\
\hline 00463-07-27 & $2.51 \times 10^{9}$ & 578 & $1.82 \times 10^{10}$ & 271 & $2.1 \times 10^{10}$ \\
\hline 00463-07-30 & $2.56 \times 10^{9}$ & 77 & $1.81 \times 10^{10}$ & 268 & $2.1 \times 10^{10}$ \\
\hline 00463-07-35 & $2.56 \times 10^{9}$ & 77 & $1.81 \times 10^{10}$ & 277 & $2.1 \times 10^{10}$ \\
\hline 00463-07-36 & $2.51 \times 10^{9}$ & 578 & $1.81 \times 10^{10}$ & 267 & $2.1 \times 10^{10}$ \\
\hline
\end{tabular}

Table 2 




Figure 1



16 corner register pixels
16 overscan register pixels

Figure 2 




Figure 3



Figure 4 


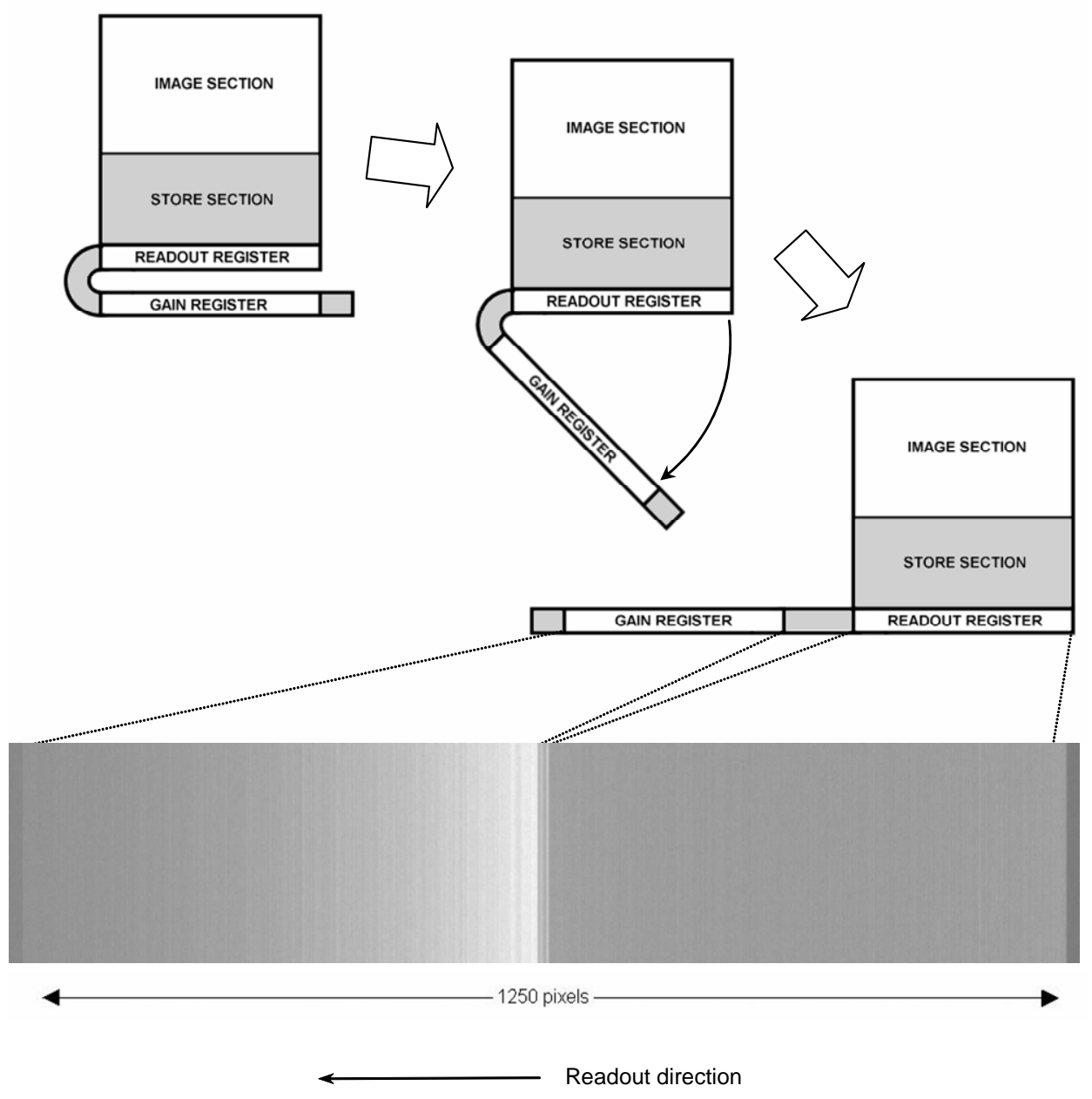

Figure 5

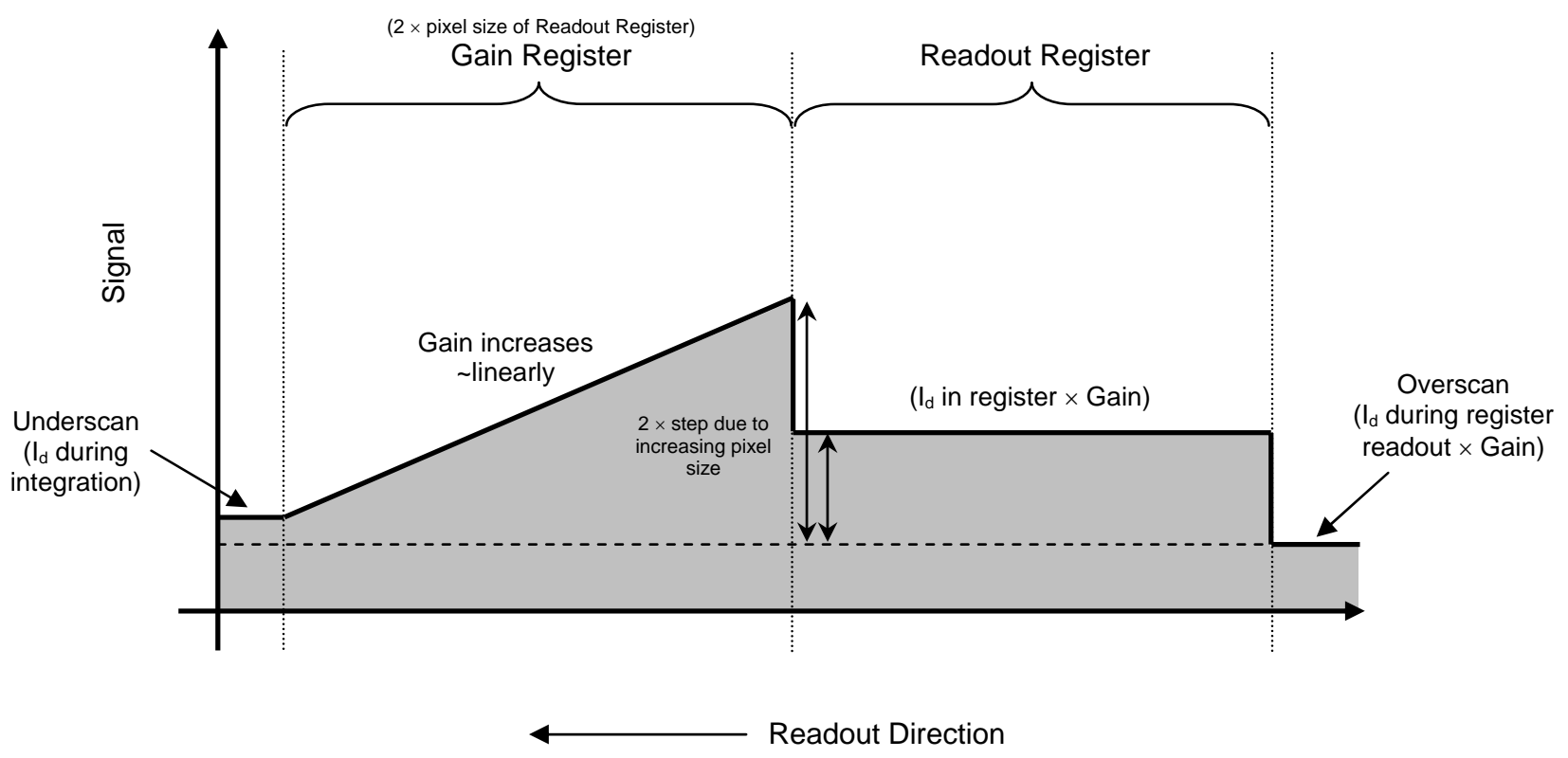

Figure 6 


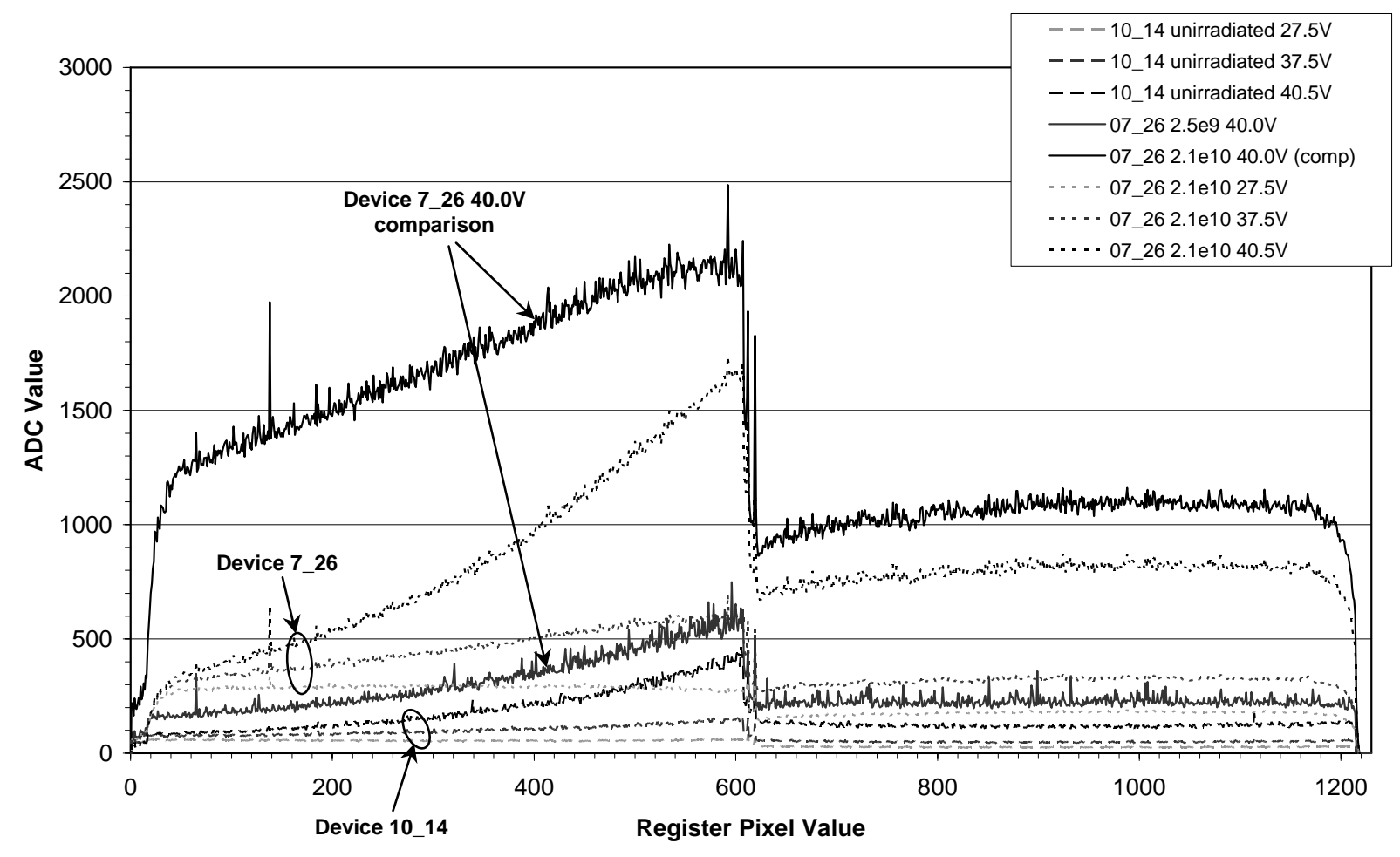

Figure 7



Figure 8 


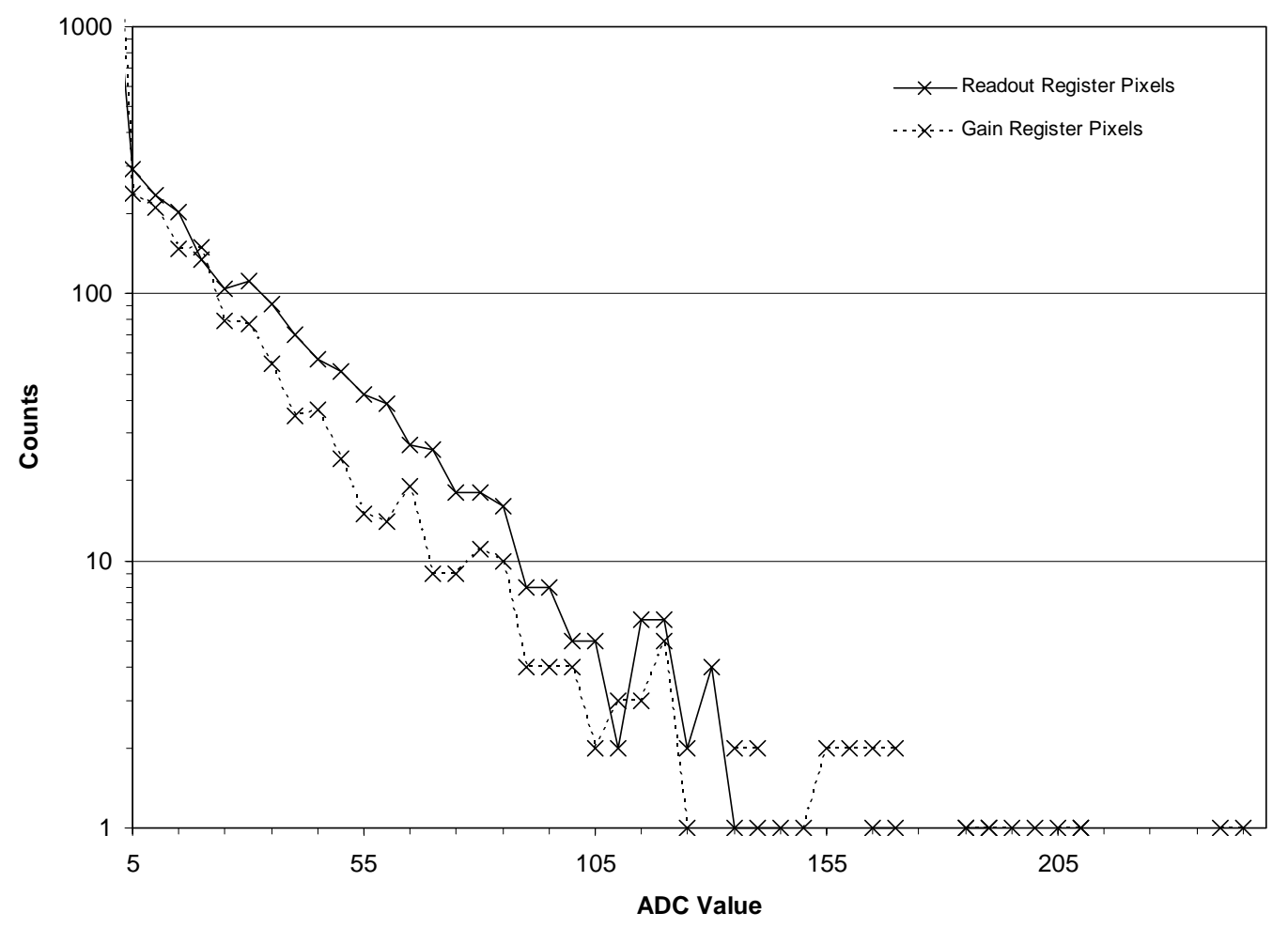

Figure 9

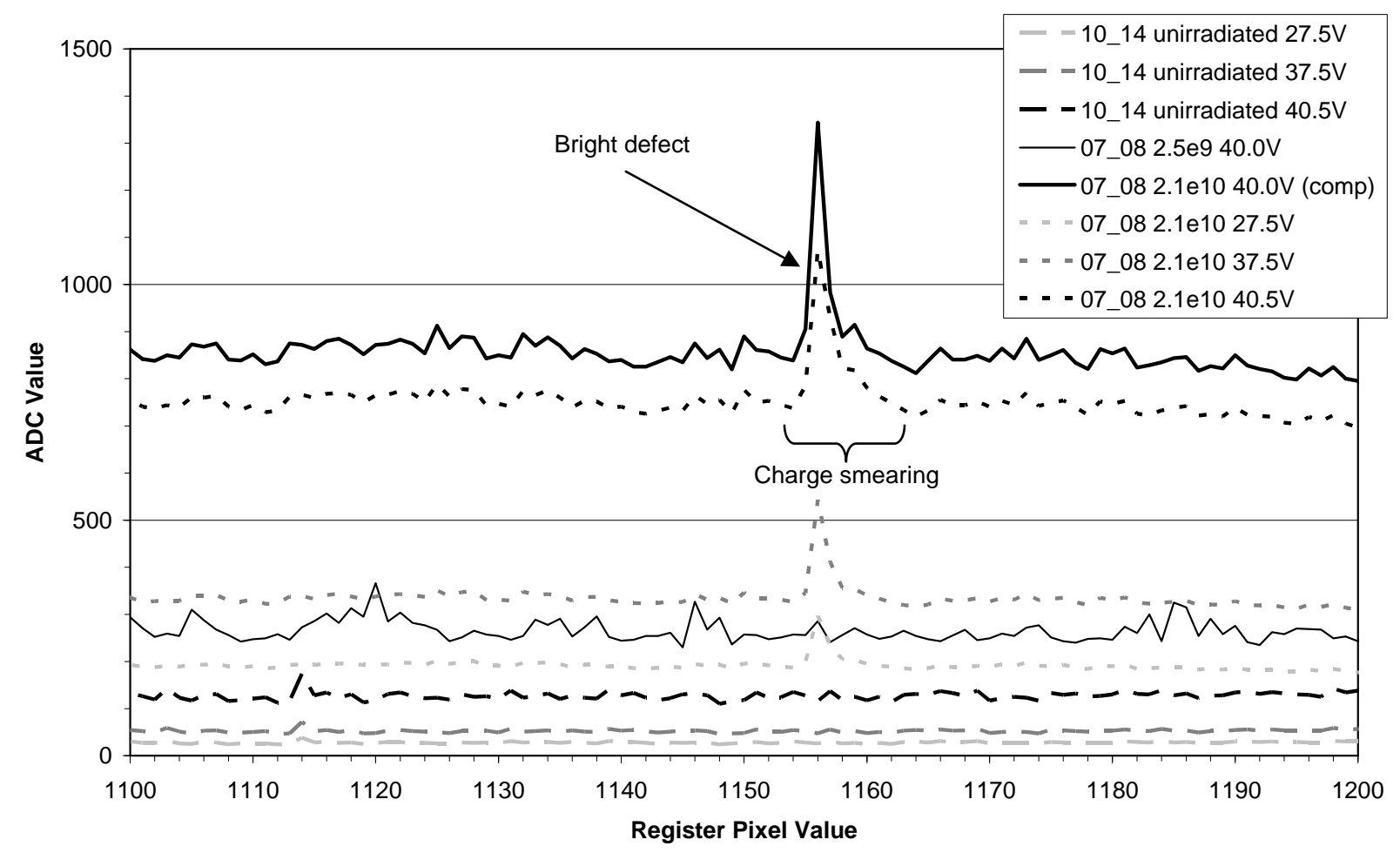

Figure 10 


\section{Tables}

Table 1: $\quad$ e2v technologies L3Vision CCD65 device parameters

Table 2: $\quad$ Irradiation parameters

\section{Figure Captions}

Figure 1: $\quad$ The e2v technologies L3Vision CCD65 device

Figure 2: $\quad$ The geometry of the e2v technologies L3Vision CCD65 device

Figure 3: $\quad$ e2v technologies measured variation of gain with applied bias voltage, $\phi \mathrm{HV}$, for an L3Vision CCD65

Figure 4: $\quad$ Irradiation geometry

Figure 5: $\quad$ A dark current image taken using a sequencer program that only reads out pixels in the readout and gain registers of the device. The readout and gain registers can clearly be seen, along with under and overscan pixels

Figure 6: $\quad$ Expected readout and gain register line profile

Figure 7: $\quad$ Unirradiated CCD65 data and data collected after each PSI irradiation for device 00463-07-26

Figure 8: $\quad$ A comparison of all 8 irradiated devices operating with $\phi \mathrm{HV}$ at $40.5 \mathrm{~V}$

Figure 9: $\quad$ Dark current spike histograms for the gain and normal readout registers of the devices after irradiation

Figure 10: $\quad$ Evidence of charge smearing in device $00463-07-08$ after irradiation with $2.1 \times 10^{10}$ protons $^{-2}$ 